\title{
Modelling and Simulation Analysis of Power Flow Router in Energy Internet
}

\author{
Lei HOU ${ }^{1}$, Kun SU${ }^{1}$, Cheng qing, ${ }^{2,}$, Tian XIA ${ }^{2}$, Chao Yang ${ }^{2}$ and Lijuan Duan ${ }^{2}$ \\ ${ }^{1}$ State Grid Xiong'an New Area Electric Power Supply Company, China \\ ${ }^{2}$ Energy Internet Research Institute, Tsinghua University, China
}

\begin{abstract}
With a large number of distributed generation (DG) and controllable load connected to the power grid under the background of energy Internet development, the security and stability of the grid are challenged. Power flow control has become a problem of the operation of the power grid. Power flow router (PFR) has become an important element of energy Internet. It is an effective way to realize the power flow control of active distribution network when abundant distributed resources are connected to the grid. In this paper, the steady-state and dynamic operation characteristics of PFR are studied, and the corresponding mathematical model is established. The effect of PFR on optimizing the power flow and improving the dynamic characteristics of distribution network is analysed by the 14-node distribution network case simulating.
\end{abstract}

\section{Introduction}

Under the background that energy Internet has become a hot topic in the field of energy research and construction at home and abroad, abundant distributed generations (DGs) and controllable loads are incorporated into the distribution network, which challenges the security and stability of the operation of the distribution network. Power flow control has become a problem of the operation of the power grid. Power flow router (PFR) has become an important element of energy Internet ${ }^{[1]}$. Following plug and play protocol, PFR can identify and manage all lowvoltage $\mathrm{DC}$ and $\mathrm{AC}$ bus connected with it, provide a variety of flexible control for controllable load, distributed energy storage system (ESS), DG, etc., and support flexible networking of various types of resources. It is an effective way to realize the power flow control of active distribution network when abundant distributed resources are connected to the grid.

The research on PFR has some foundation at home and abroad. A multi-port PFR is proposed in [2], which can dynamically control the energy flow. It embodies the nonlinear transformation and can realize the instantaneous and real-time energy transmission between multiple ports. A wireless PFR platform for controlling and scheduling energy transmission is established to minimize the peak power consumption of buildings in [3]. A multi-agent system based PFR architecture is proposed in [4]. The multi-agent system management technology is used to automatically control and coordinate the distribution network to achieve intelligent power flow routing. A general model of PFR integrated with power flow controller, various power and load interface devices and communication devices is proposed in [5] and [6]. A typical three-level modular circuit topology of distribution network PFR is presented in [7], which has three ports: high-voltage AC port, low-voltage AC port and lowvoltage DC port. In [8] and [9], a Y-type PFR based on three port isolated DC / DC converter is proposed, which uses phase-shifting technology to control the power flow in open-loop, but lacks certain generality. In [10], energy routers are classified, and a hierarchical and partitioned optimization strategy for energy Internet based on PFR is proposed. In the bottom layer, a distributed local optimization strategy is implemented with the lowest generation cost as the goal. In the upper layer, the routing and trading center adopts the transaction optimization strategy based on graph theory, and selects the lowest network loss path without blocking through PFR for power transmission to maximize benefits and congestion management. In [11] and [12], a PFR topology based on power electronic architecture is proposed, and a PFR topology applied to AC-DC hybrid microgrid is proposed in [13]. The relationship between the current effective value and the system on-state loss in the traditional phaseshift modulation mode of DC distribution network is quantitatively analysed, and the system optimal control strategy based on triangular wave current modulation is proposed. A PFR model with virtual inertia is proposed in [14], which makes the PFR not only have the characteristics of inertia and damping, but also can be applied to the constant power load of DC converter, and enhance the stability of power grid. In [15], sensitivity is integrated into optimal power flow (OPF) to increase the correction control of PFR, but the calculation is complex and the reactive power is not considered. In [16], the power flow model of PFR with the characteristics of "branch port voltage" is established, which only involves the branch power flow modelling and does not refer to the local injection power flow modelling.

*Corresponding author: chengqing@tsinghua-eiri.org 
In the above references, the topological structure and model of PFR are different, but the function description of the PFR in the power system is similar, that is, the network power flow hub integrating multiple system regulation and protection functions. In this paper, based on the above existing research on PFR, the steady-state and dynamic operation characteristics of PFR are studied, and the corresponding mathematical model is established. Then, the effect of PFR on optimizing the power flow and improving the dynamic characteristics of distribution network is analysed by the case simulating.

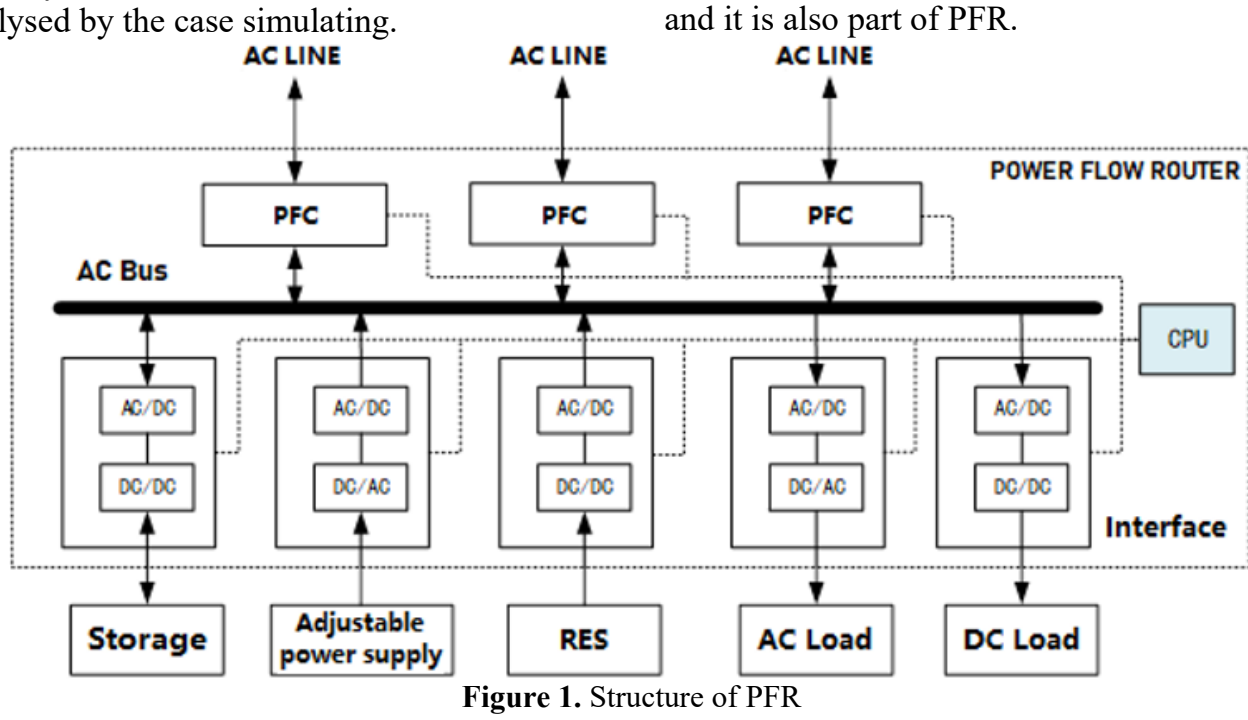

The equivalent circuit of PFC is shown in Figure 2. PFC is installed on the line connecting bus $i$ and $j$, and has three control parameters: the voltage amplitude $V_{\mathrm{S}}$ and phase angle $\phi_{\mathrm{S}}$ at the series side and the current amplitude Iq at the parallel side, which is orthogonal to bus voltage $V_{\mathrm{i}}$.



Figure 2. The equivalent circuit of PFC

According to the equivalent circuit and vector relationship, the basic mathematical relationship is as follows:

$$
\begin{aligned}
& V_{i}^{\prime}=V_{i}+V_{S} \\
& \operatorname{Arg}\left(I_{q}\right)=\operatorname{Arg}\left(V_{i}\right) \pm \pi / 2 \\
& \operatorname{Arg}\left(I_{S}\right)=\operatorname{Arg}\left(V_{i}\right) \\
& I_{S}=\frac{\operatorname{Re}\left[V_{S} I_{i}^{* *}\right]}{V_{i}}
\end{aligned}
$$

The VSC model is shown in Figure 3. The AC-DC-AC conversion is realized by $\mathrm{VSC} 1$ and $\mathrm{VSC} 2$. If the bus voltage $V_{\mathrm{i}}$ is given, the voltage phase and amplitude of $V_{\mathrm{ik}}$ can be adjusted in a certain range based on the common DC bus, so as to control the line power flow.

\section{Modelling of PFR}

The architecture of PFR is shown in Figure 1, in which all the incoming and outgoing lines are connected with the common bus through the line power flow controller (PFC), and each PFC automatically controls the corresponding line power flow. In addition, some DC or frequency conversion equipment are connected to the common bus through voltage source converter (VSC). VSC controls the output and terminal voltage of corresponding equipment, and it is also part of PFR.

AC LINE

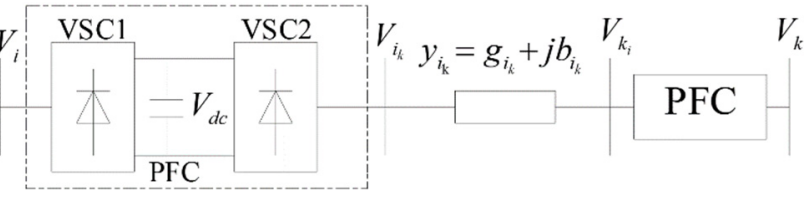

Figure 3. The equivalent circuit of VSC

$$
V_{i_{k}}=\frac{M_{2}}{M_{1}} e^{j\left(\alpha_{2}-\alpha_{1}\right)} V_{i}
$$

Where, $M_{1}$ and $M_{2}$ are the modulation ratios of VSC1 and VSC2 respectively, and $\alpha_{1}$ and $\alpha_{2}$ are the phase shift angles of VSC1 and VSC2 respectively.

From the above equivalent models, in the aspect of power flow control, the PFC and VSC can be regarded as phase-shifting transformers with controllable parameters. In addition, PFR may also include solid-state transformers and various FACTS. Therefore, PFR can be equivalent to a combination of phase-shifting transformers, series voltage sources and parallel reactive power compensation.

\subsection{Steady-state model of PFR}

The steady-state model of PFR is mainly used to solve the optimal power flow (OPF). The OPF is to find the optimal solution (system generator output, voltage amplitude and phase angle) to meet the system constraints (voltage constraints, power flow constraints, output constraints). The objective function can be composed of generation cost, network loss, transmission capacity and other indicators. Taking minimization of network loss as an example, the OPF model is as follows: 


$$
\begin{aligned}
& \min P_{b}=V_{b} \sum_{j=1}^{n} V_{j}\left(G_{b j} \cos \theta_{b j}+B_{b j} \sin \theta_{b j}\right)+P_{d b}-P_{s b} \\
& P_{s i}-P_{d i}-V_{i} \sum_{j=1}^{n} V_{j}\left(G_{i j} \cos \theta_{i j}+B_{i j} \sin \theta_{i j}\right)=0 \quad(i=1, \ldots, n) \\
& Q_{s i}-Q_{d i}-V_{i} \sum_{j=1}^{n} V_{j}\left(G_{i j} \sin \theta_{i j}-B_{i j} \cos \theta_{i j}\right)=0 \quad(i=1, \ldots, n) \\
& P_{i j}-V_{i} V_{j}\left(G_{i j} \cos \theta_{i j}+B_{i j} \sin \theta_{i j}\right)-t_{i j} V_{i}^{2} G_{i j}=0 \quad(k=1, \ldots, l) \\
& P_{j i}-V_{i} V_{j}\left(G_{i j} \cos \theta_{i j}-B_{i j} \sin \theta_{i j}\right)-V_{j}^{2} G_{i j} / t_{i j}=0 \quad(k=1, \ldots, l) \\
& V_{i \min } \leq V_{i} \leq V_{i \max } \quad(i=1, \ldots, n) \\
& P_{s i \min } \leq P_{s i} \leq P_{s i \max } \quad(i=1, \ldots, n) \\
& Q_{s i \min } \leq Q_{s i} \leq Q_{s i \max } \quad(i=1, \ldots, n) \\
& P_{i j \min } \leq P_{i j} \leq P_{i j \max } \quad(k=1, \ldots, l) \\
& P_{j i \min } \leq P_{j i} \leq P_{j i \max } \quad(k=1, \ldots, l)
\end{aligned}
$$

Where, $P$ is the active power, $Q$ is the reactive power, $V$ is the voltage amplitude, $\theta$ is the voltage phase angle, $G$ is the line conductance, $B$ is the line susceptance, and $t$ is the non-standard transformation ratio. The subscript $i$ represents the $i$ th node, $i j$ represents the line between node $i$ and node $j, s$ represents the input, $d$ represents the output, and $b$ represents the PCC point where the regional energy system connects to the superior power grid.

If PFR is considered, $t$ is regarded as a complex variable,

$$
\dot{t}=t \angle \sigma
$$

Then $G$ and $B$ are all variables of $\dot{t}$. If $t=t^{(1)}+t^{(2)} \sin \tau$, where $t^{(1)}=\left(t_{\max }+t_{\min }\right) / 2, t^{(1)}=\left(t_{\max }-t_{\min }\right) / 2$, the $G$ and $B$ are variables of $\tau$ and $\sigma$. We can rewrite (3) as the following partial differential equation:

$$
\left\{\begin{array}{l}
\frac{\partial \Delta P_{i}}{\partial \tau_{i j}}=-V_{i}\left[V_{j}\left(\frac{\partial G_{i j}}{\partial \tau_{i j}} \cos \theta_{i j}+\frac{\partial B_{i j}}{\partial \tau_{i j}} \sin \theta_{i j}\right)+V_{i} \frac{\partial G_{i i}}{\partial \tau_{i j}}\right] \\
\frac{\partial \Delta P_{i}}{\partial \sigma_{i j}}=-V_{i}\left[V_{j}\left(\frac{\partial G_{i j}}{\partial \sigma_{i j}} \cos \theta_{i j}+\frac{\partial B_{i j}}{\partial \sigma_{i j}} \sin \theta_{i j}\right)+V_{i} \frac{\partial G_{i i}}{\partial \sigma_{i j}}\right] \\
\frac{\partial \Delta Q_{i}}{\partial \tau_{i j}}=-V_{i}\left[V_{j}\left(\frac{\partial G_{i j}}{\partial \tau_{i j}} \sin \theta_{i j}-\frac{\partial B_{i j}}{\partial \tau_{i j}} \cos \theta_{i j}\right)-V_{i} \frac{\partial B_{i i}}{\partial \tau_{i j}}\right] \\
\frac{\partial \Delta Q_{i}}{\partial \sigma_{i j}}=-V_{i}\left[V_{j}\left(\frac{\partial G_{i j}}{\partial \sigma_{i j}} \sin \theta_{i j}-\frac{\partial B_{i j}}{\partial \sigma_{i j}} \cos \theta_{i j}\right)-V_{i} \frac{\partial B_{i i}}{\partial \sigma_{i j}}\right] \\
\frac{\partial \Delta f}{\partial \tau_{b j}}=-V_{b}\left[V_{j}\left(\frac{\partial G_{b j}}{\partial \tau_{b j}} \cos \theta_{b j}+\frac{\partial B_{b j}}{\partial \tau_{b j}} \sin \theta_{b j}\right)+V_{b} \frac{\partial G_{b b}}{\partial \tau_{b j}}\right] \\
\frac{\partial \Delta f}{\partial \sigma_{b j}}=-V_{b}\left[V_{j}\left(\frac{\partial G_{b j}}{\partial \sigma_{b j}} \cos \theta_{b j}+\frac{\partial B_{b j}}{\partial \sigma_{b j}} \sin \theta_{b j}\right)+V_{b} \frac{\partial G_{b b}}{\partial \sigma_{b j}}\right]
\end{array}\right.
$$

Where, $\Delta f$ is the difference between the objective function value and the upper limit value.

(5) is introduced into the nonlinear indeterminate equations of augmented constrained power flow as shown in (6). By using Newton Raphson method to solve (6), the optimal power flow with PFR can be obtained.

$$
\left[\begin{array}{c}
\Delta P \\
\Delta Q \\
\Delta f
\end{array}\right]=\left[\begin{array}{llllll}
\frac{\partial \Delta P}{\partial \theta} & \frac{\partial \Delta P}{\partial \alpha} & \frac{\partial \Delta P}{\partial \delta} & \frac{\partial \Delta P}{\partial \tau} & \frac{\partial \Delta P}{\partial \sigma} & \frac{\partial \Delta P}{\partial y_{b}} \\
\frac{\partial \Delta Q}{\partial \theta} & \frac{\partial \Delta Q}{\partial \alpha} & \frac{\partial \Delta Q}{\partial \delta} & \frac{\partial \Delta Q}{\partial \tau} & \frac{\partial \Delta Q}{\partial \sigma} & \frac{\partial \Delta Q}{\partial y_{b}} \\
\frac{\partial \Delta f}{\partial \theta} & \frac{\partial \Delta f}{\partial \alpha} & \frac{\partial \Delta f}{\partial \delta} & \frac{\partial \Delta f}{\partial \tau} & \frac{\partial \Delta f}{\partial \sigma} & \frac{\partial \Delta f}{\partial y_{b}}
\end{array}\right]\left[\begin{array}{l}
\Delta \theta \\
\Delta \alpha \\
\Delta \delta \\
\Delta \tau \\
\Delta \sigma \\
\Delta y_{b}
\end{array}\right]
$$

Where, $\alpha, \beta, \delta$ are the auxiliary decision variables of $V$, $P$ and $Q$ respectively, and $y_{\mathrm{b}}$ is the auxiliary variable that transforms the objective function into the equation form.

\subsection{Dynamic model of PFR}

PFR is mainly used in power flow and voltage control, and stability control is its auxiliary function. In order to give full play to the role of PFR in enhancing system damping, stabilizing system oscillation and enhancing system reliability, it is necessary to study the impact of adding PFR device on system transient performance. Based on the steady-state power flow control, the control strategy of PFR in the transient process is formulated by using the characteristics of PFR to quickly adjust the active and reactive power of the transmission line.

According to the structure and working principle of PFR, the transient stability model of PFR is shown in Figure 4. This model includes power control, fault crossing control and line interface. Among them, the power control link controls the transmissing active and reactive power of the line when the system is in normal operation. The fault crossing control link is the control logic when the system is subject to fault or large disturbance. The line interface takes PFR as the controlled current source and injects the calculated current into the line. The power control model and the fault crossing control model are shown in Figure 5 and Figure 6 respectively.

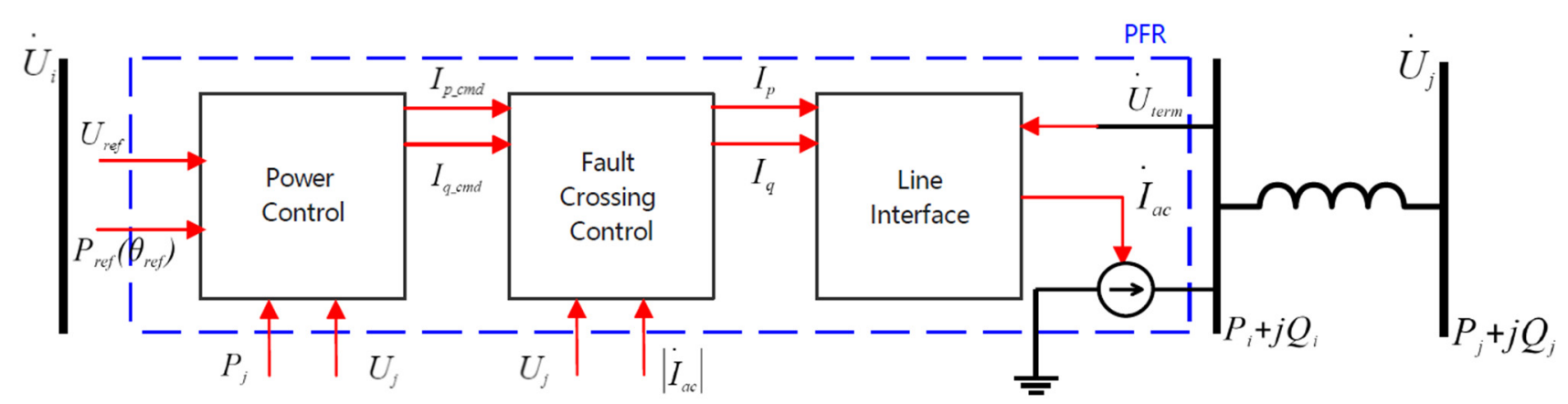

Figure 4. The transient stability model of PFR 


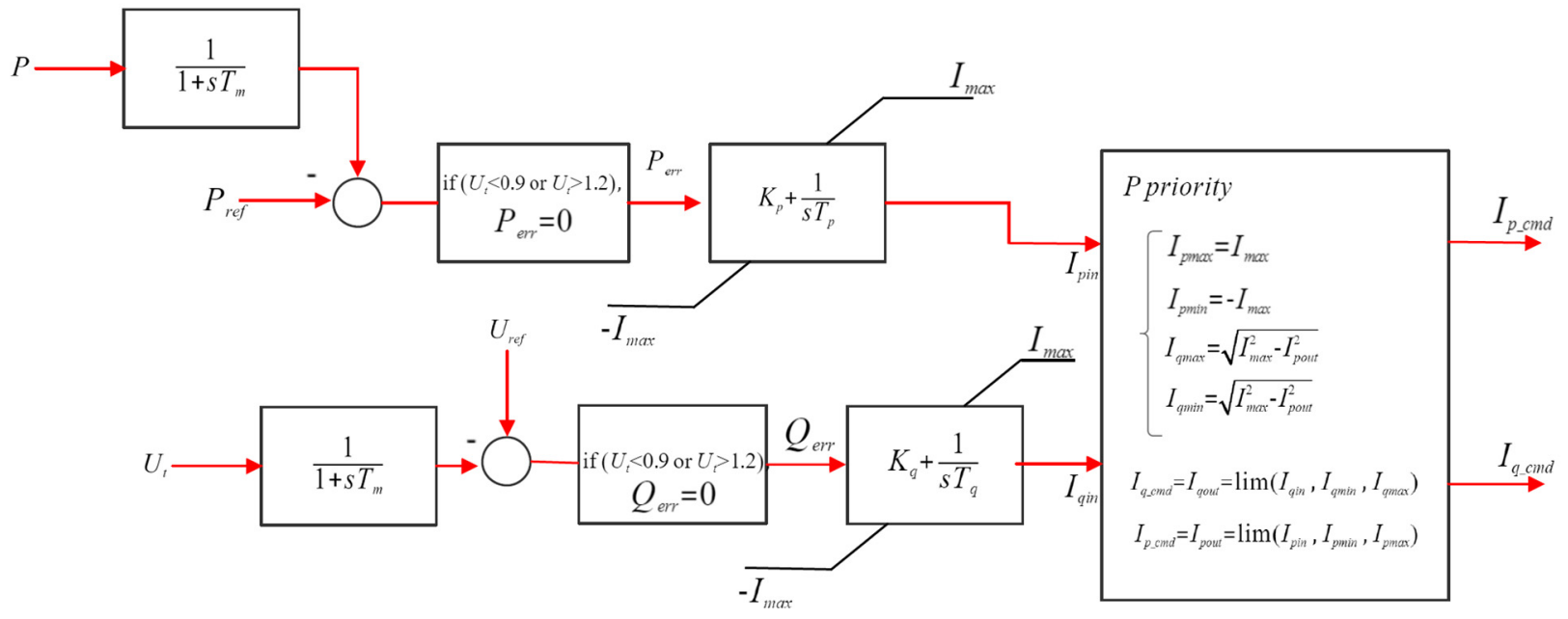

Figure 5. The power control model

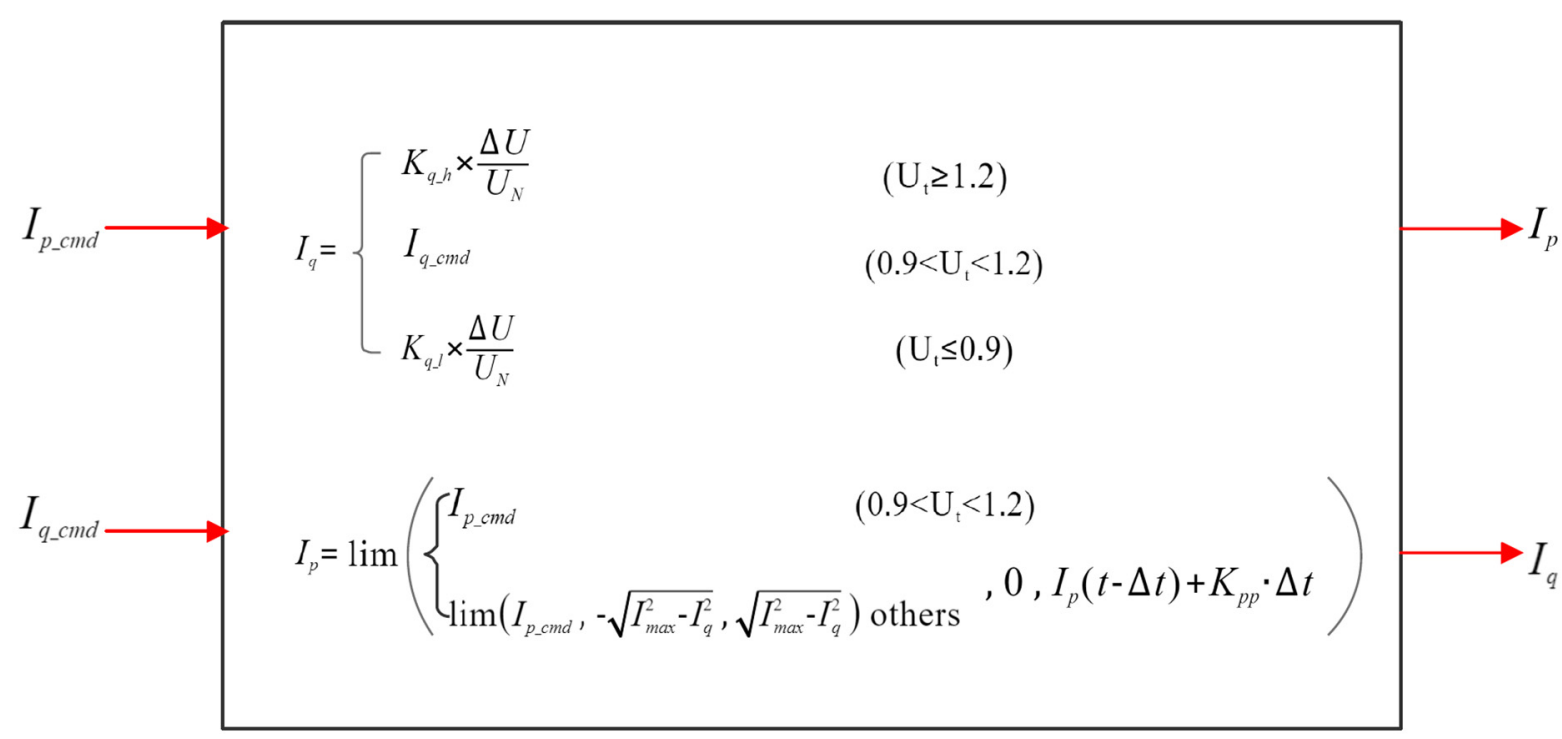

Figure 6. The fault crossing control model

Where, $T_{\mathrm{m}}$ is the measuring time constant. $I_{\max }, I_{\min }$ are maximum and minimum output current of converter respectively. $K_{\mathrm{p}}, T_{\mathrm{p}}$ are the scale factor and the integral time constant of active power control under normal working conditions. $K_{\mathrm{q}}, T_{\mathrm{q}}$ are the scale factor and the integral time constant of reactive power control under normal working conditions. $U_{\mathrm{n}}$ is the rated voltage of PFR. $K_{\mathrm{q}_{\mathrm{h}}}$ and $K_{\mathrm{q}_{1} 1}$ are high voltage and low voltage forced reactive compensation coefficient respectively. $K_{\mathrm{pp}}$ is the change rate of active current recovery.

\section{Case study}

In order to verify the above models, the IEEE standard 5 machine 14 node calculation example system is used, and the model structure is shown in Figure 7. The example system has 5 generator nodes, among which G1 is the balance node, G2, G3, G4 and G5 are PV nodes, and the other 9 nodes are PQ nodes. The system consists of 20 branches, including 3 transformer branches. The node parameters and branch parameters of the example system are shown in Table 1 and Table 2.

\subsection{Steady state simulation analysis}

In the example system, PFR is installed on the central node $\mathrm{B} 2 \mathrm{of} 220 \mathrm{kV}$ grid and the central node $\mathrm{B} 13$ of $110 \mathrm{kV}$ grid. The optimal power flow of the whole grid is solved, and the optimization objective is to minimize the network loss of the whole grid, that is, to minimize the output of the balance node G1. The comparison of calculation results is shown in Table 3. 


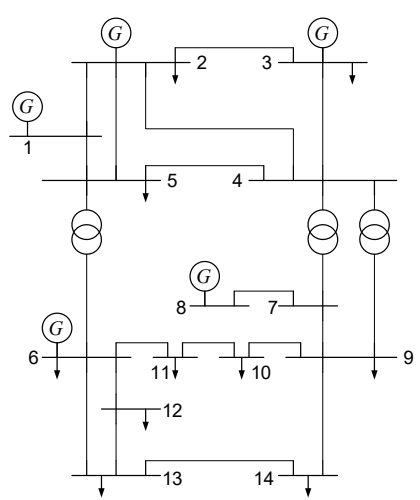

Figure 7. The fault crossing control model

Table 1. The node parameters of the example system

\begin{tabular}{ccccccc}
\hline No. & Type & $\begin{array}{c}P_{\mathrm{l}} \\
\text { (p.u.) }\end{array}$ & $\begin{array}{c}Q_{\mathrm{l}} \\
\text { (p.u.) }\end{array}$ & $\begin{array}{c}P_{\mathrm{g}} \\
\text { (p.u.) }\end{array}$ & $\begin{array}{c}Q_{\mathrm{g}} \\
\text { (p.u.) }\end{array}$ & $\begin{array}{c}V_{\mathrm{t}} \\
\text { (p.u.) }\end{array}$ \\
\hline 1 & Slack & 0 & 0 & 0 & 0 & 1.06 \\
\hline 2 & PV & 0.217 & 0.127 & 0.4 & 0 & 1.045 \\
\hline 3 & PV & 0.942 & 0.19 & 0 & 0 & 1.01 \\
\hline 4 & PQ & 0.478 & -0.039 & 0 & 0 & 1 \\
\hline 5 & PQ & 0.076 & 0.016 & 0 & 0 & 1 \\
\hline 6 & PV & 0.112 & 0.075 & 0 & 0 & 1.07 \\
\hline 7 & PQ & 0 & 0 & 0 & 0 & 1 \\
\hline 8 & PV & 0 & 0 & 0 & 0 & 1.09 \\
\hline 9 & PQ & 0.295 & 0.166 & 0 & 0 & 1 \\
\hline 10 & PQ & 0.09 & 0.058 & 0 & 0 & 1 \\
\hline 11 & PQ & 0.035 & 0.018 & 0 & 0 & 1 \\
\hline 12 & PQ & 0.061 & 0.016 & 0 & 0 & 1 \\
\hline 13 & PQ & 0.135 & 0.058 & 0 & 0 & 1 \\
\hline 14 & PQ & 0.149 & 0.05 & 0 & 0 & 1 \\
\hline
\end{tabular}

Table 2. The branch parameters of the example system

\begin{tabular}{cccccc}
\hline No. & $\begin{array}{c}\text { Connecting } \\
\text { nodes }\end{array}$ & $\begin{array}{c}R \\
\text { (p.u.) }\end{array}$ & $\begin{array}{c}X \\
\text { (p.u.) }\end{array}$ & $\begin{array}{c}B \\
\text { (p.u.) }\end{array}$ & $T_{\mathrm{k}}$ \\
\hline 1 & $1-2$ & 0.01938 & 0.05917 & 0.02640 & 1 \\
\hline 2 & $1-5$ & 0.05403 & 0.22304 & 0.02460 & 1 \\
\hline 3 & $2-3$ & 0.04699 & 0.19797 & 0.02190 & 1 \\
\hline 4 & $2-4$ & 0.05811 & 0.17632 & 0.0170 & 1 \\
\hline 5 & $2-5$ & 0.05695 & 017388 & 0.01730 & 1 \\
\hline 6 & $3-4$ & 0.06701 & 0.17103 & 0.00640 & 1 \\
\hline 7 & $4-5$ & 0.01335 & 0.04211 & 0.00000 & 1 \\
\hline 8 & $4-7$ & 0.0000 & 0.20912 & 0.00000 & 0.978 \\
\hline 9 & $4-9$ & 0.0000 & 0.55618 & 0.00000 & 0.969 \\
\hline 10 & $5-6$ & 0.0000 & 0.25202 & 0.00000 & 0.932 \\
\hline 11 & $6-11$ & 0.09498 & 0.19890 & 0.00000 & 1 \\
\hline 12 & $6-12$ & 0.12291 & 0.25581 & 0.00000 & 1 \\
\hline 13 & $6-13$ & 0.06615 & 0.13027 & 0.00000 & 1 \\
\hline 14 & $7-8$ & 0.0000 & 0.17615 & 0.00000 & 1 \\
\hline 15 & $7-9$ & 0.0000 & 0.11001 & 0.00000 & 1 \\
\hline 16 & $9-10$ & 0.03181 & 0.08450 & 0.00000 & 1 \\
\hline 17 & $9-14$ & 0.12711 & 0.27038 & 0.00000 & 1 \\
\hline 18 & $10-11$ & 0.08205 & 0.19207 & 0.00000 & 1 \\
\hline 19 & $12-13$ & 0.22092 & 0.19988 & 0.00000 & 1 \\
\hline 20 & $13-14$ & 0.17093 & 0.34802 & 0.00000 & 1 \\
\hline & & & & &
\end{tabular}

Table 3. Comparison of OPF calculating results

\begin{tabular}{cccc}
\hline No. & Work & $\begin{array}{c}\text { Output of G1 } \\
\text { (p.u.) }\end{array}$ & $\begin{array}{c}\text { Network loss } \\
\text { reduction rate }\end{array}$ \\
\hline 1 & No OPF & 2.4048 & - \\
\hline 2 & OPF without PFR & 2.3278 & $3.2 \%$ \\
\hline 3 & OPF with PFR & 2.1064 & $12.41 \%$ \\
\hline
\end{tabular}

The results show that the network loss of the optimal power flow can be reduced by $3.2 \%$ compared with that of the conventional power flow. When PFR is installed in the system, the network loss can be further reduced, which is 9.21\% lower than the optimal power flow without PFR, i.e., the installed PFR has an obvious effect on the optimal power flow of the system.

\subsection{Transient simulation analysis}

Transient simulation analysis focuses on the influence of PFR on the transient stability limit of power system. In the calculation, the dynamic model parameters of the generator, load and other equipment are selected from the dynamic model parameters of the same capacity equipment in the actual system, and the electromechanical transient simulation is used. The simulation step length is $0.01 \mathrm{~s}$, and the calculation time is $20 \mathrm{~s}$. The triggering fault of transient stability limit calculation is the N-1 fault of interconnection line of the section between the external grid and the regional system, and the time of fault occurrence is $1 \mathrm{~s}$.

The comparison of transient stability limit calculation results is shown in Table 4. Compared with the system without PFR, when PFR is installed in B2, the system load can be increased by $17.6 \mathrm{MW}$, and the transient stability limit of section power can be increased by 20.2MW. When PFR is installed in B2 and B13, the system load can be increased by $39.5 \mathrm{MW}$, and the transient stability limit of section power can be increased by $46.4 \mathrm{MW}$. It can be seen that the installation of PFR has a significant effect on improving the load capacity and transient stability limit of the system.

Table 4. Comparison of transient stability limit calculation results

\begin{tabular}{cccc}
\hline No. & Work & $\begin{array}{c}\text { System Load } \\
\text { (MW) }\end{array}$ & $\begin{array}{c}\text { Transient Stability } \\
\text { Limit (MW) }\end{array}$ \\
\hline 1 & No PFR & 268.6 & 243.3 \\
\hline 2 & PFR installed in B2 & 286.2 & 263.5 \\
\hline 3 & $\begin{array}{c}\text { PFR installed in } \\
\text { B2 and B13 }\end{array}$ & 308.1 & 289.7 \\
\hline
\end{tabular}

Based on the limit power flow mode of the system without PFR, the transient stability of the system before and after PFR installation is compared, and the simulation results are shown in Figure 8.

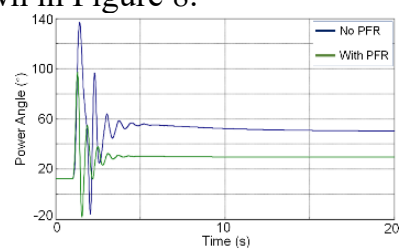

a)

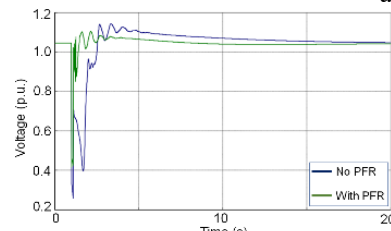

b)

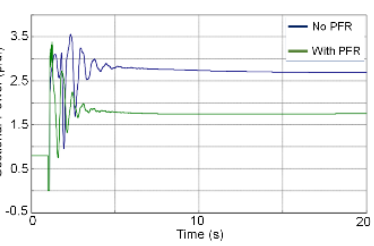

c)
Figure 8. The comparison of simulation results

It can be seen from the simulation results that when the system is installed with PFR, the power angle stability and voltage stability of the system are significantly improved when $\mathrm{N}-1$ fault occurs in the section connecting line, which is the dual effect that PFR can adjust the active and reactive power injection of the line at the same time. The transmission power deviation of the section connecting line is significantly reduced compared with that without PFR, so the stability of the system operation is improved. 


\section{Conclusions}

Power flow router (PFR) has become an important element of energy Internet. It can solve the problem of power flow control when a large number of distributed generations and controllable loads are connected into the grid. In this paper, the structure and operation principle of PFR including the line power flow controller and the voltage source converter are studied, the steady-state model of PFR based on the generalized inverse matrix method for solving OPF is established, and the transient characteristics of PFR are studied. Based on the idea of modular modelling, the general transient model of PFR is established, including power control part, fault crossing control part and line interface part.

The PFR model proposed in this paper provides an effective method for power system simulation analysis with PFR. Next, further research work will be carried out in the aspects of model improvement and actual power grid analysis, and the development of the PFR prototype will be guided.

\section{Acknowledgment}

Supported by the State Grid Corporation Headquarters Science and Technology Project (5204XQ190047)

\section{References}

1. ZHAO Zhengming, FENG Gaohui, YUAN Liqiang, ZHANG Chunpeng, 2017, "The Development and Key Technologies of Electric Energy Router", Proceedings of the CSEE, vol. 37, no. 13, 3823-3834.

2. Sánchez-Squella Antonio, Ortega Romeo, Griñó Robert, Malo Shane, 2010, "Dynamic energy router: Energy management in electrical systems fed by multiple sources", IEEE Control Systems Magazine, vol. 30 , no. $6,72-80$.

3. Behl Madhur, Aneja Mansimar, Jain Harsh, Mangharam Rahul, 2011, "EnRoute: An energy router for energy-efficient buildings", Proceedings of the 10th ACM/IEEE International Conference on Information Processing in Sensor Networks, IPSN'11, 125-126.

4. Nguyen Phuong H., Kling Wil L., Ribeiro Paulo F., 2011, "Agent-based power routing in Active Distribution Networks", 2011 2nd IEEE PES International Conference and Exhibition on Innovative Smart Grid Technologies, ISGT Europe 2011, 1-6.

5. J. Lin, V. O. K. Li, K.-C. Leung, A. Y. S. Lam, 2014, "Architectural design and load flow study of power flow routers," Proc. IEEE Int. Conf. Smart Grid
Commun. (SmartGridComm'14), 43-48.

6. Lin Junhao, Li Victor O. K., Leung Ka-Cheong, Lam Albert Y. S., 2017, "Optimal Power Flow with Power Flow Routers", IEEE Transactions on Power Systems, vol. 32, no. 1, 531-543.

7. CAO Yang, YUAN Liqiang, ZHU Shaomin, et al., 2015, "Parameter Design of Energy Router Orienting Energy Internet", Power System Technology, vol. 39, no. 11, 3094-3101.

8. Kado Yuichi, Shichijo Daiki, Deguchi Ikumi, Iwama Naoki, Kasashima Ryosuke, Wada Keiji, 2015, "Power flow control of three-way isolated DC/DC converter for Y-configuration power router", 2015 IEEE 2nd International Future Energy Electronics Conference, IFEEC 2015, 1-5.

9. Kado Yuichi, Kasashima Ryosuke, Iwama Naoki, Wada Keiji, 2016, "Implementation and performance of three-way isolated DC/DC converter using SiCMOSFETs for power flow control", 2016 IEEE 7th International Symposium on Power Electronics for Distributed Generation Systems, PEDG 2016, 1-7.

10. GUO Hui, WANG Fei, ZHANG Lijun, LUO Jian, 2016, "Technologies of Energy Router-based Smart Distributed Energy Network", Proceedings of the CSEE, vol. 36, no. 12, 3314-3324.

11. MIAO Jianqiang, ZHANG Ning, KANG Chongqing, 2017, "Analysis of the influence of energy routers on the operation optimization of distribution network", Proceedings of the CSEE, vol. 37, no. 10, 2832-2839.

12. Weijie Dong, Lijuan Hu, Wanxing Sheng, et al., 2017, "Research on probabilistic optimal power flow of distribution system with multilayer structure based on energy router", Journal of Engineering, vol. 2017, no. 13, 1621-1624.

13. TU Chunming, MENG Yang, XIAO Fan, LAN Zheng, SHUAI Zhikang, 2017, "An AC-DC Hybrid Microgrid Energy Router and Operational Modal Analysis", Transactions of China Electrotechnical Society, vol. 32, no. 22, 176-188.

14. AI Xin, TAN Qian, LV Zhipeng, ZHANG Mingze, 2018, "VSG with PBC Energy Router and Its Application in Microgrid", Journal of North China Electric Power University, vol. 45, no. 3, 1-9.

15. Thomas James Jamal, Hernandez Jorge, Grijalva Santiago, 2013, "Power flow router sensititvities for post-contingency corrective control", 2013 IEEE Energy Conversion Congress and Exposition, ECCE 2013, 2590-2596.

16. James Jamal Thomas, Jorge E. Hernandez, Santiago Grijalva, 2014, "An investigation of the impact of dispatchable power routers on electricity markets and market participants", IEEE Power and Energy Society General Meeting, vol. 2014, no. 10, 1-5. 